\title{
Vital Signs: Epidemiology of Sepsis: Prevalence of Health Care Factors and Opportunities for Prevention
}

\author{
Shannon A. Novosad, MD ${ }^{1,2}$; Mathew R.P. Sapiano, PhD²; Cheri Grigg, DVM ${ }^{1,2}$; Jason Lake, MD ${ }^{1,2}$; Misha Robyn, DVM ${ }^{1,4}$; Ghinwa Dumyati, MD 3 ; \\ Christina Felsen, $\mathrm{MPH}^{3}$; Debra Blog, $\mathrm{MD}^{4}$; Elizabeth Dufort, $\mathrm{MD}^{4}$; Shelley Zansky, $\mathrm{PhD}^{4}$; Kathryn Wiedeman, $\mathrm{MPH}^{2}$; Lacey Avery, MA ${ }^{2}$; \\ Raymund B. Dantes, $\mathrm{MD}^{2}$; John A. Jernigan, $\mathrm{MD}^{2}$; Shelley S. Magill, $\mathrm{MD}^{2}$; Anthony Fiore, $\mathrm{MD}^{2}$; Lauren Epstein, $\mathrm{MD}^{2}$
}

On August 23, 2016, this report was posted as an MMWR Early Release on the MMWR website (http://www.cdc.gov/mmwr).

\section{Abstract}

Background: Sepsis is a serious and often fatal clinical syndrome, resulting from infection. Information on patient demographics, risk factors, and infections leading to sepsis is needed to integrate comprehensive sepsis prevention, early recognition, and treatment strategies.

Methods: To describe characteristics of patients with sepsis, CDC and partners conducted a retrospective chart review in four New York hospitals. Random samples of medical records from adult and pediatric patients with administrative codes for severe sepsis or septic shock were reviewed.

Results: Medical records of 246 adults and 79 children (aged birth to 17 years) were reviewed. Overall, $72 \%$ of patients had a health care factor during the 30 days before sepsis admission or a selected chronic condition likely to require frequent medical care. Pneumonia was the most common infection leading to sepsis. The most common pathogens isolated from blood cultures were Escherichia coli in adults aged $\geq 18$ years, Klebsiella spp. in children aged $\geq 1$ year, and Enterococcus spp. in infants aged $<1$ year; for $106(33 \%)$ patients, no pathogen was isolated. Eighty-two (25\%) patients with sepsis died, including $65(26 \%)$ adults and $17(22 \%)$ infants and children.

Conclusions: Infection prevention strategies (e.g., vaccination, reducing transmission of pathogens in health care environments, and appropriate management of chronic diseases) are likely to have a substantial impact on reducing sepsis. CDC, in partnership with organizations representing clinicians, patients, and other stakeholders, is launching a comprehensive campaign to demonstrate that prevention of infections that cause sepsis, and early recognition of sepsis, are integral to overall patient safety.

\section{Introduction}

Many different infections can lead to sepsis, a serious and often fatal clinical syndrome that is characterized by organ dysfunction and can be difficult to diagnose (1-3). Sepsis is associated with high morbidity and mortality $(1-4)$ and accounted for $\$ 23.7$ billion in health care expenditures in 2013 (5). Identifying specific sepsis prevention strategies is a public health priority.

Evaluations of sepsis epidemiology have typically used death certificate or health services utilization data; these methods have well-described limitations $(6,7)$. Most sepsis initiatives have focused on improving outcomes by promoting protocol-driven approaches that facilitate early recognition and treatment (8). Detailed data regarding underlying conditions, health care factors, types of infections, and pathogens most commonly associated with sepsis could guide development of programs to inform clinicians, patients, and families about prevention of infections that can lead to sepsis. To inform sepsis initiatives and health communication efforts, CDC partnered with the New York State Department of Health and Emerging Infections Program to perform a medical record assessment to describe clinical characteristics, comorbidities, and potential opportunities for infection prevention among patients with sepsis.

\section{Methods}

A retrospective medical record review at four general, acute care hospitals in New York was performed through CDC's Emerging Infections Program. Patients were eligible for inclusion if they had a hospital admission during October 1, 2012-September 30, 2013 (fiscal year [FY] 2013), or October 1, 2014-September 30, 2015 (FY 2015). The International Classification of Diseases, Ninth Revision, Clinical Modification (ICD-9-CM) discharge diagnosis codes for severe sepsis (995.92) or septic shock (785.52) were used by hospitals to generate lists of potential cases. A target sample 
size of approximately 300 records was selected. The lists of medical records were sorted into random order, and samples of records were selected and reviewed to identify demographic characteristics, underlying conditions, and infections leading to sepsis. Patients' demographic and clinical characteristics were abstracted using a standardized form. Patients whose medical records did not include documentation of sepsis and patients for whom large portions of the medical record were incomplete or missing were excluded.

The first date of clinical documentation of sepsis or a related term in the chart by a clinician was used to classify sepsis cases. The timing of first sepsis documentation was used as a proxy for sepsis onset, and the presence of health care factors prior to the sepsis hospitalization was used to classify cases as community- or health care-associated. Cases were classified as community-associated/community-onset if the initial documentation of sepsis occurred at admission or during the first 3 calendar days of admission, with the date of admission considered to be day 1 , and if, during the 30 days preceding admission, there were no health care factors (i.e., $\geq 2$ days in a nursing home, long-term or other acute care hospital, receipt of intravenous antimicrobials, peritoneal or hemodialysis, surgery, total parenteral nutrition, chemotherapy, wound therapy, or presence of a central venous catheter). Community-associated/ community-onset cases were further categorized based on whether the patient had one or more of a number of selected chronic conditions that might require frequent contact with health care providers for management.*

Sepsis cases were classified as health care-associated if the initial documentation of sepsis occurred $\leq 3$ days after admission and health care factors were identified during the preceding 30 days; they were further categorized as nursing home-onset, community-onset, or undetermined-onset, depending on the location from which the patient was admitted to the hospital. Sepsis cases for which the first documentation of sepsis occurred after day 3 of admission were classified as health care-associated/hospital-onset. A descriptive analysis of demographics, clinical characteristics, underlying chronic conditions, pathogens, and infection types among patients with sepsis was performed.

\section{Results}

Adult patients with sepsis. Charts of 290 adult patients with sepsis were selected, and reviews were completed for 246 (85\%); 44 (15\%) records were excluded, most commonly

\footnotetext{
* Selected chronic conditions included any of the following: steroid/ immunosuppressive therapy, acquired immunodeficiency syndrome, cerebral palsy, hemiplegia, spinal cord injury, paraplegia, quadriplegia, congestive heart failure, chronic obstructive pulmonary disease, cystic fibrosis, chronic ventilator/ tracheostomy, leukemia, lymphoma, multiple myeloma, neutropenia, transplant (hematopoietic and solid organ), solid tumor (metastatic and not metastatic), sickle cell disease, cirrhosis, or diabetes mellitus with complications.
}

because encounter information was missing. The median age of adult patients with sepsis was 69 years; 127 (52\%) were male (Table 1). The median length of hospital stay was 9 days. Most patients (238 [97\%]) had at least one comorbidity; $87(35 \%)$ had diabetes mellitus, 79 (32\%) had cardiovascular disease (including coronary artery disease, peripheral vascular disease, or congestive heart failure), 56 (23\%) had chronic kidney disease, and 50 (20\%) had chronic obstructive pulmonary disease. The most common illnesses leading to sepsis were pneumonia (85 [35\%]), urinary tract infections (62 [25\%]), gastrointestinal infections (28 [11\%]), and skin/ soft tissue infections (26 [11\%]) (Table 2). Pathogens were isolated from blood cultures of $75(30 \%)$ patients and from urine cultures of $70(28 \%)$; these groups were not mutually exclusive. The most common pathogens identified from blood were Staphylococcus spp. (including both S. aureus and coagulase negative Staphylococcus), Escherichia coli, and Streptococcus spp. (Table 3). For 76 (31\%) patients with sepsis, no pathogen was identified in any culture or nonculture based tests.

Among the 246 adult patients with sepsis whose records were reviewed, $142(58 \%)$ were classified as health care-associated with $44(18 \%)$ of these hospital-onset and 104 (42\%) were classified as community-associated, without health care factors. Among health care-associated sepsis cases, the most common health care factors were acute care hospitalization or nursing home stays of $\geq 2$ days in the preceding 30 days, which were reported for $44(18 \%)$ and $43(17 \%)$ patients, respectively. Among patients with community-associated sepsis, nearly $50 \%$ ( $20 \%$ of all patients with sepsis) had a selected chronic condition likely to lead to frequent encounters with health care providers, such as diabetes mellitus with complications, cancer, or congestive heart failure. Pneumococcal vaccination before the sepsis hospitalization was documented for 108 (44\%) patients, and influenza vaccination in the year before admission was documented for 87 (35\%) patients.

Among the 155 patients admitted from a private residence, $23(15 \%)$ were discharged to a long-term care facility. Sixty-five (26\%) patients died during their sepsis hospitalization, including 47 (representing $72 \%$ of deaths) who were aged $\geq 65$ years, and seven (representing $11 \%$ of deaths) who had no health care factors in the 30 days preceding admission.

Pediatric patients with sepsis. Records of 88 pediatric patients with sepsis were selected for review, and reviews were completed for 79 (90\%), including 31 infants aged $<1$ year (39\%), and 48 children, aged 1-17 years (61\%) (Table 1). At least one comorbidity was present for $62(78 \%)$ pediatric patients, including 25 (81\%) infants and 37 (77\%) children. The most common comorbidity among infants was congenital heart disease, affecting seven (23\%) patients; the most common comorbidities among children were cognitive deficits or cerebral palsy, affecting 18 (38\%). 
TABLE 1. Number and percentage of sepsis cases among adult $(N=246)$ and pediatric $(N=79)$ patients, by selected characteristics - four acute care hospitals, New York, fiscal years 2013 and 2015

\begin{tabular}{|c|c|c|c|}
\hline \multirow[b]{2}{*}{ Characteristic } & \multirow{2}{*}{$\begin{array}{l}\text { Adult patients } \\
(\mathrm{N}=246)^{*} \\
\text { No. }(\%)\end{array}$} & \multicolumn{2}{|c|}{ Pediatric patients $(\mathrm{N}=79)^{*}$} \\
\hline & & $\begin{array}{l}<1 \text { year, }(n=31) \\
\text { No. }(\%)\end{array}$ & $\begin{array}{l}\geq 1 \text { year, }(n=48) \\
\text { No. }(\%)\end{array}$ \\
\hline Male sex & $127(52)$ & $22(71)$ & $26(54)$ \\
\hline Age: median, (Q1, Q3) (years) & $69(60,81)$ & - & $12(7,15)$ \\
\hline Hospital stay: median, (Q1, Q3) (days) & $9(5,18)$ & $19(7,37)$ & $11(7,19)$ \\
\hline Influenza vaccine received in year preceding sepsis (yes) ${ }^{\dagger}$ & $87(35)$ & $1(3)$ & $10(21)$ \\
\hline Pneumococcal vaccine received (yes) & $108(44)$ & $2(6)$ & $14(29)$ \\
\hline Comorbidities (any) & $238(97)$ & $25(81)$ & $37(77)$ \\
\hline Comorbidities (selected)§ & $149(61)$ & $1(3)$ & $16(33)$ \\
\hline Health care-associated/Hospital-onset ${ }^{\mathfrak{\imath}, * *}$ & $44(18)$ & $12(39)$ & $8(17)$ \\
\hline Health care-associated/Nursing home-onset $t^{\natural,}+\dagger$ & $34(14)$ & - & $1(2)$ \\
\hline Health care-associated/Community-onset ${ }^{\natural, \S \S}$ & $53(22)$ & $1(3)$ & $8(17)$ \\
\hline 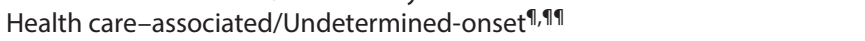 & $11(4)$ & $2(6)$ & $2(4)$ \\
\hline $\begin{array}{l}\text { Community-associated/Community-onset, no health care factors, with } \\
\text { selected comorbidities*** }\end{array}$ & $50(20)$ & $1(3)$ & $7(15)$ \\
\hline $\begin{array}{l}\text { Community-associated/Community-onset, no health care factors, without } \\
\text { selected comorbidities }{ }^{\dagger+\dagger}\end{array}$ & $54(22)$ & $15(48)$ & $22(46)$ \\
\hline \multicolumn{4}{|l|}{ Preadmission location } \\
\hline Private residence & $155(63)$ & $6(19)$ & $34(71)$ \\
\hline Nursing home/SNF & 44 (18) & - & $2(4)$ \\
\hline Other acute care hospital & $24(10)$ & $14(45)$ & $12(25)$ \\
\hline Other/Unknown & $23(9)$ & $11(35)$ & - \\
\hline \multicolumn{4}{|l|}{ Discharge disposition } \\
\hline Private residence & $102(41)$ & $17(55)$ & $38(79)$ \\
\hline Nursing home/SNF & $61(25)$ & $1(3)$ & $4(8)$ \\
\hline Other acute care hospital & $2(<1)$ & - & $1(2)$ \\
\hline Other/Unknown & $2(<1)$ & $1(3)$ & - \\
\hline Hospice & $14(6)$ & - & - \\
\hline Died during hospitalization & $65(26)$ & $12(39)$ & $5(10)$ \\
\hline
\end{tabular}

Abbreviations: Q1 = 25th percentile; Q3 = 75th percentile; SNF = skilled nursing facility.

${ }^{*}$ Adult and pediatric populations were not sampled proportionally.

† Vaccination in year prior to sepsis diagnosis.

$\S$ Steroid/immunosuppressive therapy, acquired immunodeficiency syndrome, cerebral palsy, hemiplegia, spinal cord injury, paraplegia, quadriplegia, congestive heart failure, chronic obstructive pulmonary disease, cystic fibrosis, chronic ventilator/tracheostomy, leukemia, lymphoma, multiple myeloma, neutropenia, transplant (hematopoietic and solid organ), solid tumor (metastatic and not metastatic), sickle cell disease, cirrhosis, or diabetes mellitus with complications.

" Health care-associated cases included any of the following in the preceding 30 days: $\geq 2$ days in a nursing home, long-term acute care hospital or other acute care hospital, intravenous antimicrobials, peritoneal or hemodialysis, surgery, total parenteral nutrition, central venous catheter, wound therapy, or onset after 3 days in the hospital.

** First documentation of sepsis $>3$ days after admission.

t+ Sepsis documented within 3 days of admission; patient was admitted from a nursing home and spent at least 2 calendar days in a nursing home during the preceding 30 days.

$\S \S$ Sepsis documented within 3 days of admission; patient was admitted from private residence but had a health care factor in the preceding 30 days.

१ศ Sepsis documented within 3 days of admission; patient was admitted from another hospital or nursing home and had one other health care exposure (excludes those who were both admitted from a nursing home and spent at least 2 calendar days in a nursing home during preceding 30 days).

** Sepsis documented within 3 days of admission; patient had no health care factors in preceding 30 days, with selected comorbidities (steroid/immunosuppressive therapy, acquired immunodeficiency syndrome, cerebral palsy, hemiplegia, spinal cord injury, paraplegia, quadriplegia, congestive heart failure, chronic obstructive pulmonary disease, cystic fibrosis, chronic ventilator/tracheostomy, leukemia, lymphoma, multiple myeloma, neutropenia, transplant (hematopoietic and solid organ), solid tumor (metastatic and not metastatic), sickle cell disease, cirrhosis, or diabetes mellitus with complications.

${ }^{t+\dagger}$ Sepsis documented within 3 days of admission; patient had no health care factors in preceding 30 days, without selected comorbidities.

Among pediatric patients, 34 (43\%) had sepsis with health care factors, and $45(57 \%)$ were community-associated sepsis cases without health care factors (Table 1). The most commonly identified health care factor, receipt of intravenous antibiotics in the 30 days preceding sepsis admission, was reported for eight $(10 \%)$ patients. Selected chronic conditions (Table 1) likely to require frequent medical care were identified in eight (10\%) community-associated cases.

Among infections leading to sepsis, respiratory infections were most common, and preceded sepsis in $29 \%$ of all pediatric patients, followed by gastrointestinal infections (24\%) (Table 2). Among 41 (52\%) patients for whom a pathogen was identified in a blood culture, Enterococcus spp. and Klebsiella spp. were most commonly identified in infants (14\%) and children (9\%), respectively (Table 3). In 30 (38\%) pediatric patients, sepsis was diagnosed but no pathogen was isolated. Seventeen $(22 \%)$ pediatric cases died during their sepsis hospitalization, including 12 (39\%) infants and five (10\%) children. 
TABLE 2. Number and percentage of types of infections among adult $(\mathrm{N}=246)$ and pediatric $(\mathrm{N}=79)$ patients with sepsis - four acute care hospitals, New York, fiscal years 2013 and 2015

\begin{tabular}{|c|c|c|c|}
\hline \multirow[b]{2}{*}{ Type of infection } & \multirow[b]{2}{*}{$\begin{array}{l}\text { Adult patients } \\
\begin{array}{c}(\mathrm{N}=246)^{*} \\
\text { No. }(\%)\end{array}\end{array}$} & \multicolumn{2}{|c|}{ Pediatric patients $(\mathrm{N}=79)$} \\
\hline & & $\begin{array}{l}<1 \text { year, } \\
(n=31) \\
\text { No. }(\%)\end{array}$ & $\begin{array}{l}\geq 1 \text { year, } \\
(n=48) \\
\text { No. }(\%)\end{array}$ \\
\hline Respiratory tract ${ }^{\dagger}$ & $86(35)$ & $9(29)$ & $14(29)$ \\
\hline Urinary tract & $62(25)$ & $1(3)$ & $4(8)$ \\
\hline Gastrointestinal $^{\S}$ & $28(11)$ & $8(26)$ & $11(23)$ \\
\hline Unknown/Undetermined" & $32(13)$ & $6(19)$ & $8(17)$ \\
\hline Skin and soft tissue & $26(11)$ & $1(3)$ & $3(6)$ \\
\hline Bloodstream & $13(5)$ & $8(26)$ & $6(13)$ \\
\hline Bone and joint & $6(2)$ & $1(3)$ & $1(2)$ \\
\hline Cardiovascular & $5(2)$ & $2(6)$ & - \\
\hline Eye/Ear/Nose/Throat & $3(1)$ & - & $2(4)$ \\
\hline Central nervous system & $2(<1)$ & $1(3)$ & $2(4)$ \\
\hline Disseminated systemic viral & $2(<1)$ & - & $1(2)$ \\
\hline Surgical site & $2(<1)$ & - & - \\
\hline None documented & $22(9)$ & - & $2(4)$ \\
\hline \multirow{7}{*}{\multicolumn{4}{|c|}{$\begin{array}{l}\text { * Patients can have more than one type of infection. Adult and pediatric } \\
\text { populations were not sampled proportionally. } \\
+ \text { Includes upper respiratory infections and pneumonia. Upper respiratory } \\
\text { infections in one }(<1 \%) \text { adult, three }(33 \%)<1 \text { year, and zero }(0 \%) \geq 1 \text { year. } \\
\text { Pneumonia in } 85(99 \%) \text { adults, six }(67 \%)<1 \text { year, and } 14(100 \%) \geq 1 \text { year. } \\
\S \text { Includes intra-abdominal, gastrointestinal tract, Clostridium difficile, and } \\
\text { hepatobiliary infections. Intra-abdominal infections in } 10(36 \%) \text { adults, four }(50 \%) \\
<1 \text { year, and seven }(64 \%) \geq 1 \text { year. Gastrointestinal tract infections in five }(18 \%) \\
\text { adults, four }(50 \%)<1 \text { year, and three }(27 \%) \geq 1 \text { year. Clostridium difficile infections } \\
\text { in seven }(25 \%) \text { adults, } 0(0 \%)<1 \text { year, and one }(1 \%) \geq 1 \text { year. Hepatobiliary } \\
\text { infections in six }(21 \%) \text { adults, zero }(0 \%)<1 \text { year, and zero }(0 \%) \geq 1 \text { year. }\end{array}$}} \\
\hline & & & \\
\hline & & & \\
\hline & & & \\
\hline & & & \\
\hline & & & \\
\hline & & & \\
\hline
\end{tabular}

\section{Conclusions and Comments}

The findings from this analysis will inform expansion of efforts by CDC and partners to describe the epidemiology of sepsis, prevent infections that lead to sepsis, and educate clinicians and patients about reducing the risk for sepsis. Patients with sepsis experience severe illness and serious adverse outcomes, including long hospital stays (median $=10$ days), discharge to long-term care settings (20\%), and death (25\%). Similar to other studies $(1,9,10)$, sepsis most commonly occurred among patients with one or more comorbidities, and a majority of patients developed infections leading to sepsis outside a hospital. Among all patients with sepsis, $72 \%$ had either a health care factor in the month preceding admission or a chronic condition likely to require frequent contact with the health care system, suggesting that opportunities exist for prevention or earlier recognition of infections leading to sepsis. Although multiple infections and organisms among patients with sepsis were identified in this study and in others $(2,11,12)$, in many cases a specific pathogen is not determined.

Because different types of infections can lead to sepsis, many interventions that are currently viewed as pathogen-specific or disease-specific should also be considered opportunities to prevent sepsis and included in efforts to improve sepsis education. For example, pneumonia is the most common infection causing
TABLE 3. Number and percentage of types of pathogens commonly isolated* from blood cultures of adult $(\mathrm{N}=225)$ and pediatric $(\mathrm{N}=75)$ patients with sepsis - four acute care hospitals, New York, fiscal years 2013 and $2015^{\dagger}$

\begin{tabular}{|c|c|c|c|}
\hline \multirow[b]{2}{*}{ Type of pathogen } & \multirow[b]{2}{*}{$\begin{array}{l}\text { Adult patients } \\
(\mathrm{N}=225) \\
\text { No. }(\%)^{\S}\end{array}$} & \multicolumn{2}{|c|}{ Pediatric patients $(\mathrm{N}=75)$} \\
\hline & & $\begin{array}{l}<1 \text { year } \\
(n=29) \\
\text { No. }(\%)^{\S}\end{array}$ & $\begin{array}{l}\geq 1 \text { year } \\
(n=46) \\
\text { No. }(\%)^{\S}\end{array}$ \\
\hline Escherichia coli & $17(8)$ & $2(7)$ & $3(7)$ \\
\hline Streptococcus spp.? & $15(7)$ & $2(7)$ & $2(4)$ \\
\hline $\begin{array}{l}\text { Coagulase negative } \\
\text { Staphylococcus spp. }\end{array}$ & $14(6)$ & $1(3)$ & - \\
\hline Staphylococcus aureus & $13(6)$ & $3(10)$ & $1(2)$ \\
\hline Pseudomonas spp. & $3(1)$ & $1(3)$ & $1(2)$ \\
\hline Bacillus spp. & $3(1)$ & - & - \\
\hline Enterococcus spp. & $3(1)$ & $4(14)$ & $1(2)$ \\
\hline Enterobacter spp. & $3(1)$ & - & - \\
\hline Candida spp. & $1(<1)$ & $1(3)$ & $1(2)$ \\
\hline Klebsiella spp. & $1(<1)$ & - & $4(9)$ \\
\hline
\end{tabular}

* Only pathogens isolated from more than two patients (adult and pediatric patients combined) are shown.

+ Pathogens isolated from cultures collected from 7 days before through 2 days after the first sepsis documentation in the medical record.

$\S$ Denominator includes all patients with blood cultures (positive or negative); patients could have more than one blood culture or pathogen isolated from the blood.

I Streptococcus spp. (not further speciated) 7 patients, Streptococcus pneumoniae 3 patients, Streptococcus Group B 3 patients, Streptococcus Group A 3 patients, Streptococcus Group D 2 patients.

sepsis $(2,11,12)$, and vaccination is an important and highly effective prevention strategy. Pneumococcal and influenza vaccination have both been shown to have saved thousands of lives, despite suboptimal vaccination coverage in the U.S. population; thousands more deaths could be prevented with better coverage (13-16).

Among those patients for whom sepsis onset was determined, $79.4 \%$ were classified as having sepsis onset outside of the hospital (i.e., first medical record documentation of sepsis at admission or in the first 3 hospital days). The majority of patients in this analysis had recent interactions with the health care system before admission. While this likely reflects the vulnerability of chronically ill patients to infection, it also suggests that health care facilities and providers could play a central role in sepsis prevention by providing age-appropriate and condition-appropriate vaccination to all patients and optimizing the health status of patients with chronic conditions. In addition, facility-level interventions are available to reduce the risk for health care-associated infections, such as appropriate hand hygiene and personal protective equipment to limit pathogen spread among patients. The potential impact of facility-level interventions is demonstrated through awareness and prevention efforts focused on infections caused by central line-associated bloodstream infections (CLABSIs). During 1990-2010, it is likely that as many as 198,000 CLABSIs were prevented in intensive care units, attributable at least 
in part to the successful and widespread implementation of evidence-based CLABSI prevention measures (17). Finally, efforts to reduce exposure to antibiotic-resistant organisms and disruption of the protective normal microbiome should also be included among sepsis prevention strategies.

Sepsis prevention activities led by CDC and partners are focused on five key areas: 1) increasing sepsis awareness among patients, families, and providers and building a coalition of clinical professional partners and patient advocates to work with CDC; 2) promoting early recognition of sepsis and aligning antibiotic stewardship efforts with early recognition; 3) identifying at-risk populations for prevention and early recognition efforts; 4) developing better sepsis surveillance methods to measure the impact of interventions; and 5) preventing infections that lead to sepsis, including infections caused by antibiotic-resistant pathogens. These efforts are being coordinated with partners to reduce sepsis risk, improve patient outcomes, and track progress. Recent sepsis initiatives focused on early recognition and treatment include legislation passed in 2014 in New York requiring hospitals to report a variety of sepsis process measures to the New York State Department of Health, and the Centers for Medicare \& Medicaid Services implementation in 2015 of a new policy instrument to improve sepsis care increasing the use of specific and timely medical interventions during the first few critical hours that patients with sepsis are in the hospital or emergency room (18). In addition, the Society for Critical Care Medicine and the European Society of Intensive Care Medicine recently published guidelines intended to improve early recognition of sepsis (3).

The findings in this report are subject to at least five limitations. First, the assessment examined medical records from a small sample of patients and hospitals; characteristics of patients with sepsis could be different elsewhere, although these results are consistent with previous studies. Second, a sample of adult and pediatric records were reviewed, and the numbers of records are not proportional to the actual number of adult and pediatric patients with sepsis in these facilities. Third, to identify patients with sepsis and septic shock, administrative codes were used along with confirmation that at least one provider had documented sepsis in the medical record, rather than application of an objective definition based on physiological or laboratory criteria. Therefore, although this approach has obvious limitations, it reflects the clinical impression of treating providers. Fourth, because this analysis relied on medical records for all information, data might be incomplete. Information on outpatient clinic visits was not collected; therefore, the proportion of patients with sepsis who have health care factors before their sepsis hospitalizations might have been underestimated. Finally, in many patients more than one infectious process was present, and it is possible that not all of the infections and organisms described actually caused sepsis in an individual patient.

Sepsis is a significant public health and clinical management challenge. CDC continues to work closely with numerous clinical professional organizations and patient advocates and will partner with stakeholders to launch a comprehensive campaign targeting clinicians and the public, demonstrating how steps to prevent and urgently recognize sepsis are critical components of patient safety programs. Routine health care encounters should be used as opportunities to implement interventions that could reduce the risk for infections leading to sepsis. These interventions include increasing vaccination coverage, educating patients and families about early sepsis warning signs, improving infection control programs, and optimizing chronic disease management. In addition, current efforts led by CDC and partners to improve sepsis surveillance will enhance analysis of risk factors and infections leading to sepsis and provide a more objective measure to track trends and evaluate interventions, informing overall prevention, recognition, and treatment efforts.

\section{Acknowledgments}

Donna Kent, Albany Medical Center, Sarah Elmendorf, Albany Medical Center, Nancy Spina, New York State Department of Health, and Foster Gesten, New York State Department of Health Office of Quality and Patient Safety. The four New York hospitals that participated in this assessment: University of Rochester Medical Center, Rochester General Hospital, Highland Hospital, and Albany Medical Center.

\begin{tabular}{l}
\hline${ }^{1}$ Epidemic Intelligence Service, CDC; ${ }^{2}$ Division of Healthcare Quality \\
Promotion, National Center for Emerging and Zoonotic Infectious Diseases, \\
CDC; ${ }^{3}$ Emerging Infections Program, University of Rochester Medical Center, \\
Rochester, New York; ${ }^{4}$ New York State Department of Health. \\
Corresponding author: Lauren H. Epstein, lepstein@cdc.gov, 404-639-8162.
\end{tabular}

\section{References}

1. Cohen J, Vincent JL, Adhikari NK, et al. Sepsis: a roadmap for future research. Lancet Infect Dis 2015;15:581-614. http://dx.doi.org/10.1016/ S1473-3099(15)70112-X

2. Angus DC, Linde-Zwirble WT, Lidicker J, Clermont G, Carcillo J, Pinsky MR. Epidemiology of severe sepsis in the United States: analysis of incidence, outcome, and associated costs of care. Crit Care Med 2001;29:1303-10. http://dx.doi.org/10.1097/00003246-200107000-00002

3. Singer M, Deutschman CS, Seymour CW, et al. The third international consensus definitions for sepsis and septic shock (Sepsis-3). JAMA 2016;315:801-10. http://dx.doi.org/10.1001/jama.2016.0287

4. Martin GS, Mannino DM, Eaton S, Moss M. The epidemiology of sepsis in the United States from 1979 through 2000. N Engl J Med 2003;348:1546-54. http://dx.doi.org/10.1056/NEJMoa022139

5. Torio CM, Moore BJ. National inpatient hospital costs: the most expensive conditions by payer, 2013. HCUP statistical brief no. 204. Rockville, MD: Agency for Healthcare Research and Quality; May 2016. http://www. hcup-us.ahrq.gov/reports/statbriefs/sb204-Most-Expensive-HospitalConditions.pdf 
6. Gaieski DF, Edwards JM, Kallan MJ, Carr BG. Benchmarking the incidence and mortality of severe sepsis in the United States. Crit Care Med 2013;41:1167-74. http://dx.doi.org/10.1097/CCM.0b013e31827c09f8

7. Epstein L, Dantes R, Magill S, Fiore A. Varying estimates of sepsis mortality using death certificates and administrative codes-United States, 1999-2014. MMWR Morb Mortal Wkly Rep 2016;65:342-5. http://dx.doi.org/10.15585/mmwr.mm6513a2

8. Society of Critical Care Medicine. Surviving sepsis campaign. Surviving sepsis campaign bundles. Mount Prospect, IL: Society of Critical Care Medicine; 2015. http://www.survivingsepsis.org/Bundles/Pages/ default.aspx

9. Seymour CW, Liu VX, Iwashyna TJ, et al. Assessment of clinical criteria for sepsis. For the third international consensus definitions for sepsis and septic shock (Sepsis-3). JAMA 2016;315:762-74. http://dx.doi. org/10.1001/jama.2016.0288

10. McCormick K. National trends of severe sepsis and septic shock among Centers for Medicaid \& Medicare Services beneficiaries using administrative claims data, incidence and mortality, 2008. Presented at the annual Society for Healthcare Epidemiology of America Spring Conference, Atlanta, GA, May 18-21, 2016.

11. Ani C, Farshidpanah S, Bellinghausen Stewart A, Nguyen HB. Variations in organism-specific severe sepsis mortality in the United States: 1999-2008. Crit Care Med 2015;43:65-77. http://dx.doi.org/10.1097/ CCM.0000000000000555

12. Lagu T, Rothberg MB, Shieh MS, Pekow PS, Steingrub JS, Lindenauer PK. Hospitalizations, costs, and outcomes of severe sepsis in the United States 2003 to 2007. Crit Care Med 2012;40:754-61. http://dx.doi. org/10.1097/CCM.0b013e318232db65
13. Moore MR, Link-Gelles R, Schaffner W, et al. Effect of use of 13-valent pneumococcal conjugate vaccine in children on invasive pneumococcal disease in children and adults in the USA: analysis of multisite, population-based surveillance. Lancet Infect Dis 2015;15:301-9. http:// dx.doi.org/10.1016/S1473-3099(14)71081-3

14. Williams WW, Lu PJ, O'Halloran A, et al. Surveillance of vaccination coverage among adult populations_-United States, 2014. MMWR Surveill Summ 2016;65(No. SS-1). http://dx.doi.org/10.15585/mmwr.ss6501a1

15. Foppa IM, Cheng PY, Reynolds SB, et al. Deaths averted by influenza vaccination in the U.S. during the seasons 2005/06 through 2013/14. Vaccine 2015;33:3003-9. http://dx.doi.org/10.1016/j. vaccine.2015.02.042

16. CDC. FluVaxView: influenza vaccination coverage: 2010-11 through 2014-15 state, regional, and national vaccination trend report. Atlanta, GA: US Department of Health and Human Services, CDC; 2015. http:// www.cdc.gov/flu/fluvaxview/reportshtml/trends/index.html

17. Wise ME, Scott RD 2nd, Baggs JM, et al. National estimates of central line-associated bloodstream infections in critical care patients. Infect Control Hosp Epidemiol 2013;34:547-54. http://dx.doi. org/10.1086/670629

18. National Quality Forum. Severe sepsis and septic shock: management bundle (NQF 0500). Washington, DC: National Quality Forum; 2015. http://emcrit.org/wp-content/uploads/2015/06/0500.pdf 